\title{
e-Phaïstos
}

e-Phaïstos

Revue d'histoire des techniques / Journal of the history

of technology

VII-1 | 2019

Le travail humain

\section{Les rites dans la transmission et la pérennisation des savoir-faire céramiques chez les Gwa d'Oguédoumé (sud côtier de la Côte d'Ivoire)}

Rites in the Transmission and Sustainability of Ceramic Know-How for Gwaof Oguédoumé (Southern Coastal of the Ivory Coast)

\section{Siméon Kouakou KOUASSI}

\section{OpenEdition}

Journals

Édition électronique

URL : http://journals.openedition.org/ephaistos/4555

DOI : 10.4000/ephaistos.4555

ISSN : 2552-0741

Éditeur

IHMC - Institut d'histoire moderne et contemporaine (UMR 8066)

Référence électronique

Siméon Kouakou KOUASSI, « Les rites dans la transmission et la pérennisation des savoir-faire céramiques chez les Gwa d'Oguédoumé (sud côtier de la Côte d'Ivoire) », e-Phaïstos [En ligne], VII-1 | 2019, mis en ligne le 06 avril 2019, consulté le 20 avril 2019. URL : http:// journals.openedition.org/ephaistos/4555; DOI : 10.4000/ephaistos.4555

Ce document a été généré automatiquement le 20 avril 2019

Tous droits réservés 


\title{
Les rites dans la transmission et la pérennisation des savoir-faire céramiques chez les Gwa d'Oguédoumé (sud côtier de la Côte d'Ivoire)
}

\author{
Rites in the Transmission and Sustainability of Ceramic Know-How for Gwaof \\ Oguédoumé (Southern Coastal of the Ivory Coast)
}

Siméon Kouakou KOUASSI

\section{Introduction}

1 A travers l'histoire de l'humanité, on constate une forte récurrence du rite dans les sociétés humaines ${ }^{1}$. Tout se passe comme si l'homme, imitant l'action du créateur divin, a créé des objets abstraits et des objets matériels ${ }^{2}$ pour réguler son quotidien. Nous voudrons ici nous interroger sur l'importance de ces rites dans la société $\mathrm{Gwa}^{3}$ (cf. Figure 1), une population du sud côtier et forestier de Côte d'Ivoire, et leurs incidences sur la production céramique. Cela, en vue de comprendre la pérennisation de ce savoir-faire dans une région où l'on constate la disparition à grande échelle de la poterie traditionnelle.

2 L'étude s'appuie sur des enquêtes auprès des potières d'Oguédoumé en pays Gwa. Les séances de travail avec les potières se sont voulues les plus ouvertes possible en vue de nous permettre de recueillir le maximum d'informations. Ce, dans la mesure où, nous nous trouvons devant les dernières dépositaires de ce savoir-faire en zone forestière de Côte d'Ivoire. La vulgarisation de la vaisselle en aluminium de même qu'en plastique, et le désintérêt manifesté par les jeunes filles pour la relève, ayant précipité cette situation constatée depuis $1968^{4}$. Pour ce faire, nous avons gardé le contact avec les populations 
après une enquête préalable menée au mois d'août 2011. Nous nous sommes également appuyé sur la chefferie, qui, comprend le bien fondé de la valorisation du patrimoine et des savoir-faire traditionnels surtout quand ceux-ci sont en voie de disparition. Elle s'est attelée à la sensibilisation des potières à notre enquête, afin de les amener à nous donner les informations que nous recherchions. Nous nous sommes entretenus individuellement avec chaque potière dans sa concession, sur son lieu de production.

Figure 1 : La zone d'étude

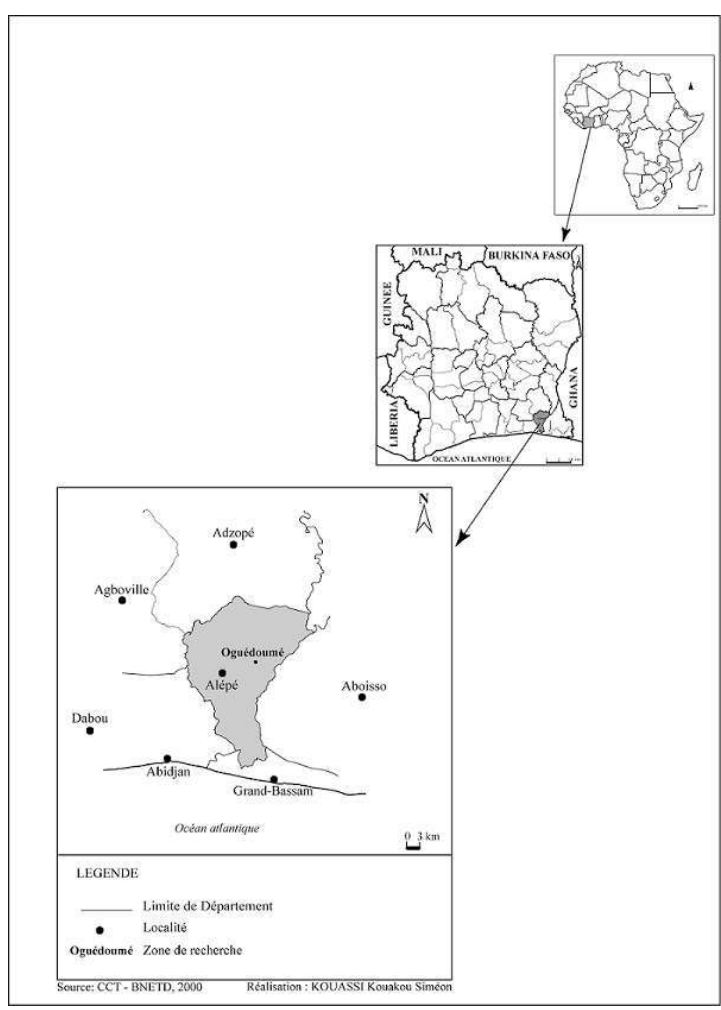

Oguédoumé de par sa situation géographique se trouve au cœur du pays Gwa. II traduit des liens séculaires avec les peuples Akyé (Alépé et Adzopé) et Agni Sanwi (Aboisso) qui dominent le sud-est ivoirien.

Source : CCT-BNETD. 2000. Réalisation : KOUASSI Kouakou Siméon

3 Nous avons ainsi pu procéder aux enregistrements d'usage à savoir : prise de notes et photographies pour l'archivage. Nous avons proscrit l'enregistrement au magnétophone parce que contrairement aux usages, sa présence bloque souvent l'information que la personne veut véhiculer et il crée une barrière artificielle entre la personne qui interroge et celle qui est interrogée. Dans notre cas le bénéficie c'est que les femmes de façon naturelle ont pu parler sans pression. Un certain nombre d'informations a pu être recueilli.

Nous montrons premièrement, les représentations de la matière première surtout plastique dans la production céramique dans le contexte africain en vue de cerner quelques subtilités de la question que nous traitons. Deuxièmement, nous présentons le panorama des rites qui organisent l'univers des potières Gwa. Troisièmement, nous analysons les interférences des rites dans la production de la céramique dans cette aire culturelle. 


\section{Représentations de la terre et ou de l'argile dans la cosmogonie africaine en général}

5 La terre, considérée comme puissance féminine et maternelle, est perçue comme le réceptacle de la puissance totale qui vient du ciel par l'entremise de l'eau, de la lumière et de l'obscurité. Considérée comme Mère des êtres, le ciel devient son époux, la lune son astre, le soleil son pôle. Elle est le giron, le dos et la mamelle maternelle des êtres. Le culte de la terre est la base même du culte animiste. C'est dans cette matrice, ou sur elle, ou sous elle, que se constituent les règnes $d u \operatorname{cosmos}^{5}$. Son importance se perçoit dans la complexité des relations politiques qui relient le village à l'ensemble dont il relève. La terre permet de même de cerner les relations politiques stricto sensu à travers la reconnaissance d'une autorité étatique territoriale ${ }^{6}$.

6 La terre en outre permet de comprendre le monde chez l'africain ${ }^{7}$ qui se subdivise en deux sphères: l'une, visible et l'autre invisible occupée par des êtres désignés généralement par le terme "génie " qui animent les différents éléments de l'univers ${ }^{8}$. Si le ciel est la demeure de l'être suprême, la terre elle, représente l'espace occupé par les êtres vivants composés des hommes, des animaux, des eaux, des plantes. Au sein du monde visible, l'Africain ancien distingue deux espaces : un socialisé ou culturel habité par l'homme et l'autre non socialisé ou naturel composé entre autres par les montagnes, les forêts denses, les îles, les eaux, les marécages, les rapides, les profondeurs et les hauteurs, habitacle des dieux intermédiaires (Orisha des Yoruba, Busson des Agni) plus ou moins bienveillants.

7 De ce point de vue on poursuivra avec Gadou' pour constater que la religion traditionnelle africaine [...], apparaît être une religion de l'alliance éternelle entre l'homme et la nature par la médiation des génies, des ancêtres, des Dieux. L'homme africain ancien en symbiose avec cette nature y note une force contingente, vu qu'il y reconnaît des puissances qui sont à la fois bienfaisantes et malveillantes. Signe pour lui que la nature est chargée de signifiants et de signes du cosmos, porteurs de messages significatifs auxquels on s'allie pour sécuriser le quotidien. La nature, de même que les éléments qui la composent, est ainsi perçue comme lieu de résidence des ancêtres à partir duquel ils surveillent les agissements des vivants ${ }^{10}$. A partir de la religion traditionnelle, par exemple les Ehotilé, un peuple de l'extrême Sud-est de la Côte d'Ivoire, contrôle, en grande partie, les systèmes sociaux ${ }^{11}$.

8 Aussi, ressort-il dans le monde Africain ancien, que le fait communautaire découle d'une conception de la vie fondée sur la recherche d'un équilibre harmonieux au sein du groupe qui place dans une même dynamique la société, la nature et le sacré. Ainsi pour ces peuples, l'homme qui vit en harmonie avec la nature entre dans les grâces de la divinité12. Elément fondamental du patrimoine collectif, la terre apparait ainsi comme une source de vie pour les membres du groupe pour les ressources qu'elle offre pour les cultures, les pâturages, les mines, la faune, la flore. Ainsi, sa perte ou son amputation pour des faits de guerre ou son hostilité perceptible à travers la sécheresse, la désertification, l'inondation ${ }^{13}$ est toujours mal ressentie par les gardiens des traditions qui par la divination cherchent les raisons profondes de telles situations en interrogeant les mânes des ancêtres. Le culte des ancêtres est ainsi perçu comme un moment grave rattaché très souvent à la découverte de l'agriculture ${ }^{14}$. La terre chez les peuples ruraux africains, est ainsi liée à l'organisation sociale et économique, et présente dans ce contexte, un lien fort 
chez les populations paysannes. La terre y représente la base même de la survie du groupe.

9 L'agriculture et l'élevage sont les deux grands métiers des animistes. On sacrifie à la terre, car de sa fécondité dépend celle de tout l'univers. Aussi chaque village avait-il son sacrificateur, appelé "gérant de la terre ». L'agriculteur ne sème ni ne plante avant de demander à la terre d'accepter tout d'abord, puis de veiller sur la transformation de la graine qu'il lui confie. Il lui demande pardon avant de la fendre avec sa houe, afin qu'elle accepte cette blessure sans colère. Le travail des champs étant considéré comme un processus de procréation, c'est pourquoi, en certains endroits, ce sont les hommes qui fendent la terre, tandis que seules les femmes sont habilitées à enfouir la graine dans le sein de la terre comme en une matrice, en raison de leur parenté analogique avec elle. En effet, les secrets profonds de la vie sont cachés dans les entrailles de la terre et dans ses excavations. On notera ainsi chez certains peuples le développement de certaines approches divinatoires pour s'allier la force que représente la terre.

Il en est ainsi de la géomancie, une technique divinatoire par la terre pratiquée quotidiennement par un très grand nombre d'ethnies d'Afrique noire de l'Ouest mais aussi d'Asie mineure, d'Afrique du Nord, et du Nord-Est, de Madagascar et des Comores ${ }^{15}$. Dans ce jeu de redistribution du matériel ethnologique, les sachant réorganisent par des gestes et des paroles un appareil formel implicite, invariant bien que mathématiquement malléable pour traduire des opérations de type sacrificiel liées à une divination par la terre. De ce fait, et pour que la terre réponde favorablement aux requêtes des vivants son intégrité doit être préservée.

11 Ainsi en Côte d'Ivoire au sortir de la période dite de la crise post-électorale (2010-2011) qui entraîna des décès en masse, la Commission Dialogue, Vérité et Réconciliation (CDVR) a initié une période de deuil des violences et de purification pour faire, à l'échelle nationale, le deuil des violences et des tragédies qui ont frappé le pays depuis plus de dix ans. En effet, la compréhension d'une telle initiative ${ }^{16}$ relève du fait que le patrimoine culturel africain attache un prix particulier à la vie. En conséquence, toute effusion de sang entraînant mort d'homme est vécue comme une catastrophe dans la mesure où, elle conduit à la rupture de trois liens sacrés que sont la relation à la terre forcée d'absorber le sang humain versé, le respect envers les esprits tutélaires profanés et le lien du défunt avec Dieu et l'Univers. Rétablir donc cet équilibre du monde perturbé par le crime de sang exige l'observation d'un rituel de purification conformément aux us et coutumes du terroir. Ce rituel, dans les sociétés traditionnelles africaines, a le pouvoir psychologique d'atténuer les tensions sociales. Il favorise la recherche de la vérité et de la réconciliation en débarrassant la société de ses impuretés spirituelles consécutives au crime de sang. A cette occasion, la communauté prend l'engagement de prôner la paix.

\section{Panorama des usages qui organisent l'univers des potières Gwa}

Les pratiques qui organisent l'univers des potières Gwa se sont perpétuées, comme dans la plupart des cas, à travers des initiations assidues. L'initiation consiste à transmettre à un individu une science, un art ainsi qu'une profession à travers l'ensemble des cérémonies par lesquelles on était admis à la connaissance de certains « mystères ». 
13 L'apprentissage de la profession de potière chez les Gwa est libre. Il n'y a pas de dispositions particulières à observer ${ }^{17}$. La poterie s'apprend dans cette aire culturelle en observant sa mère à l'œuvre. Cette dernière l'ayant apprise de sa mère. Une chaine d'apprentissage est ainsi tissée et ne s'interrompra que si un maillon saute dans le jeu du legs, gage de la perpétuation des savoir-faire.

14 Dans le détail, la transmission du savoir se fait dans le secret de l'atelier familial. Au début de l'initiation, à partir de son plus jeune âge (entre 9 ans et 10 ans), la future potière fabrique de petits vases. La mère en plus de ses vases convoie au marché ceux de l'apprentie potière. Le jour où l'entièreté de la production du néophyte est entièrement écoulée sur le marché, est retenu comme un signe d'intérêt et de bon apprentissage. La mère dès cet instant, passe à une autre étape en enseignant à la fille d'autres techniques de façon graduelle en produisant des récipients de plus en plus volumineux résultant de méthodes qui nécessitent un savoir-faire dont la difficulté s'accentue progressivement. Les bases du métier sont ainsi apprises au fur et à mesure.

15 L'apprentissage apparait de ce point de vue comme une véritable école avec des passages de grade. BOUAME Youa Félicité, potière à Oguédoumé en pays Gwa, a ainsi appris les rudiments du métier pendant une dizaine d'année auprès de sa mère à qui elle succéda suite à son décès. Elle vouait ainsi une obéissance presqu'aveugle aux us et coutumes de son groupe social. Elle révèle que par le passé, les jeunes filles s'intéressaient à la poterie, ce qui n'est plus le cas aujourd'hui. D'où la disparition programmée de l'activité, comme susmentionné, avec la dernière génération de potière qu'elle représente, avec ses collègues de même génération du village. En effet, les potières visitées sont toutes bientôt septuagénaires (cf. Photo $\mathrm{n}^{\circ} 1$ et $\mathrm{n}^{\circ} 2$ ), et surtout malades, d'où la perte de vitesse de l'activité.

16 Le métier de potière est exclusivement réservé aux femmes. Les hommes ne s'y adonnent pas même pour la cueillette de l'argile qui est un travail qui demande une force physique souvent importante. A cela s'ajoute l'observation d'interdits que nous qualifions de rites de «propreté ». 


\section{Photo $\mathrm{n}^{\circ} 1$ : Potières d'Oguédoumé BOUAME Youa}

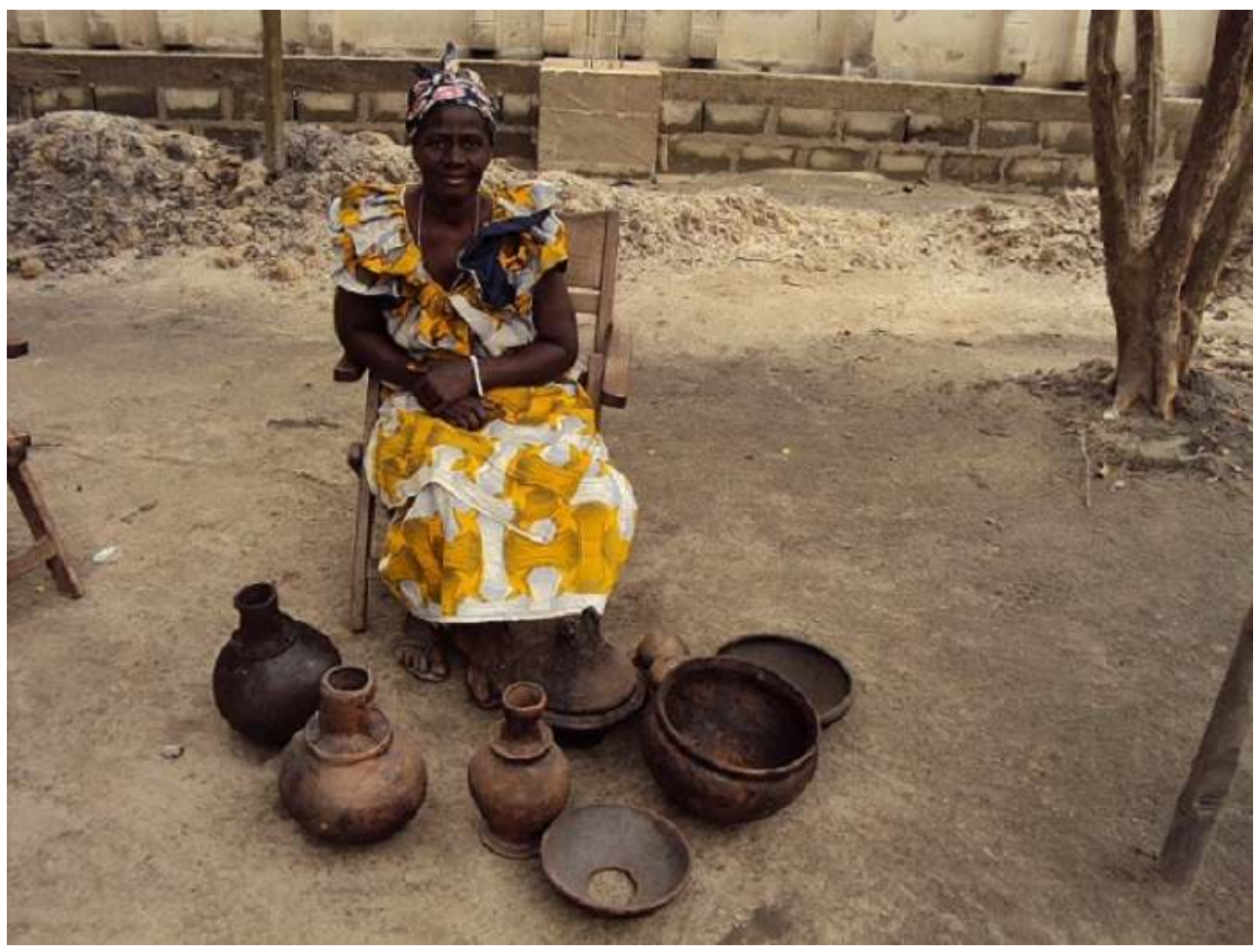

La poterie dans la zone forestière et côtière (sud) de la Côte d'Ivoire est remarquable par l'âge avancé des potières. Comparativement à la région des savanes (nord) les femmes qui détiennent ce savoirfaire à Oguédoumé sont en nombre réduit. Les rares femmes qui pratiquent le métier, de façon occasionnelle, sont toutes septuagenaires. C'est le cas de KOFFI Atoua et de BOUAME Youa que nous avons eu le bonheur d'interroger. Leur ouverture d'esprit a permis de collecter des données importantes sur l'art de la terre cuite dans cette partie de la Côte d'Ivoire. En se rendant disponibles, elles nous ont permis d'inscrire la céramique Gwa, sur la carte de cette technique pour le territoire ivoirien.

Credits : Koukou Siméon KOUASSI 


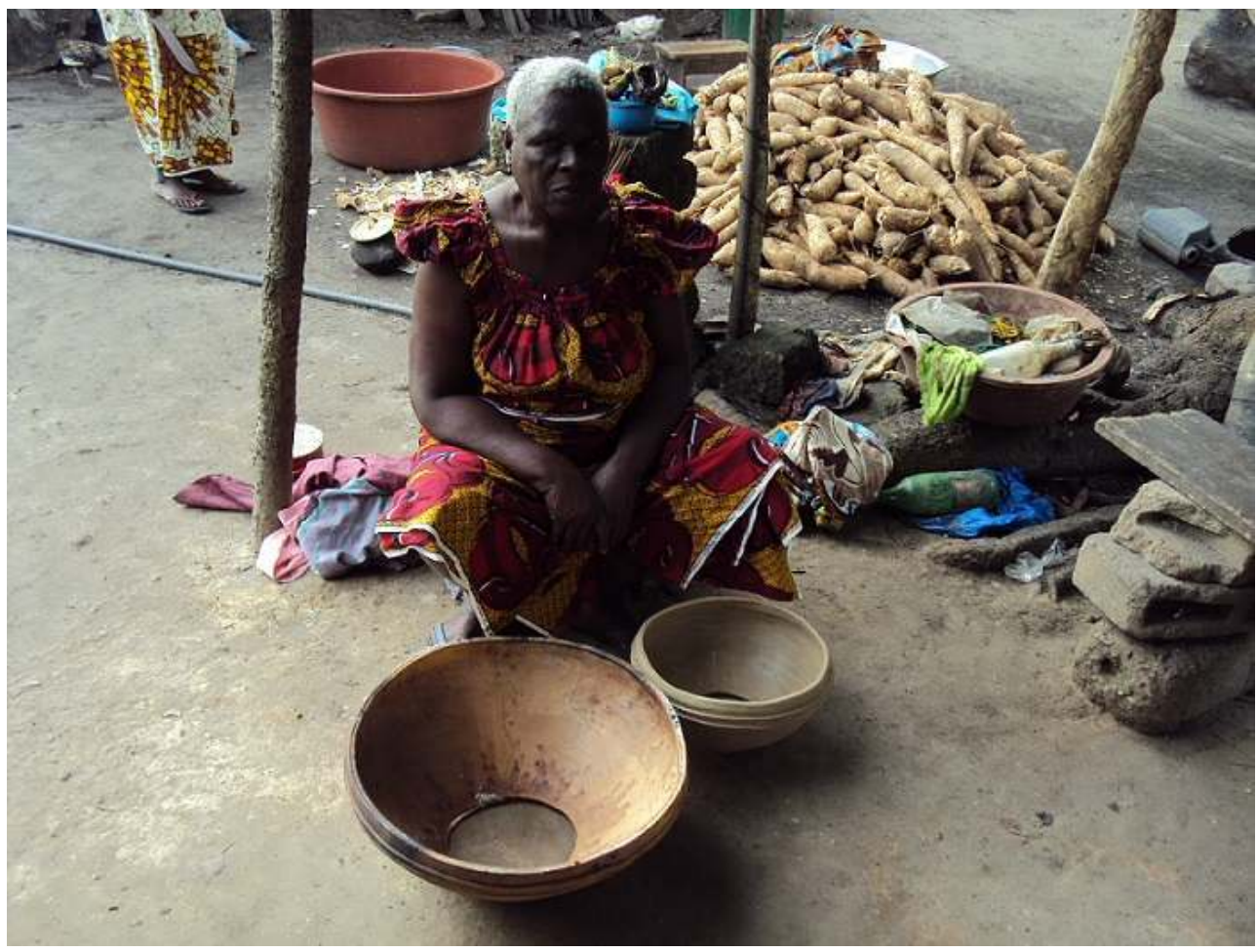

La poterie dans la zone forestière et côtière (sud) de la Côte d'Ivoire est remarquable par l'âge avancé des potières. Comparativement à la région des savanes (nord) les femmes qui détiennent ce savoirfaire à Oguédoumé sont en nombre réduit. Les rares femmes qui pratiquent le métier, de façon occasionnelle, sont toutes septuagenaires. C'est le cas de KOFFI Atoua et de BOUAME Youa que nous avons eu le bonheur d'interroger. Leur ouverture d'esprit a permis de collecter des données

importantes sur l'art de la terre cuite dans cette partie de la Côte d'Ivoire. En se rendant disponibles, elles nous ont permis d'inscrire la céramique Gwa, sur la carte de cette technique pour le territoire ivoirien.

Credits : Koukou Siméon KOUASSI

\section{Rites associés aux phases de production céramique à Oguédoumé}

17 Les rites dits de propreté ${ }^{18}$ sont de trois types. D'abord, il est formellement interdit à une femme qui a ses menstrues de se rendre dans les carrières et d'extraire l'argile, ni produire des poteries. Les menstrues dans les sociétés traditionnelles sont considérées comme une matière dangereuse. La crainte du sang remonte, comme nous l'enseigne Makarius $^{19}$ à la chasse, à l'affrontement des bêtes sauvages, et à l'adoption de stratégies pour surmonter la peur qu'elle suscite, le rapport entre les blessures infligées aux animaux, l'épandage de sang, l'agonie et la mort. Le sang qui s'écoule est ainsi synonyme de présage des dangers et rappelle les maux auxquels sont confrontés les humains. C'est une matière qui effraie et son seul contact est destructeur. En évitant le contact et la vue du sang, en bannissant ce qui peut en évoquer l'idée et en éloignant les personnes qui en sont souillées ou qui saignent, les hommes pensent ainsi se prémunir des dangers et maux qu'ils appréhendent. Pour la pensée l'impureté n'est que le danger du sang et par extension ce qui lui est associé, ou le suggère. La terre qui en elle-même est sacrée, parce que siège des ancêtres, ne doit pas être forcée à absorber du sang au risque de la souiller 
et provoquer la colère de ces derniers, qui pourraient jeter de mauvais sorts aux vivants en leur infligeant des maladies surnaturelles ${ }^{20}$. La maladie prend alors le sens d'une sanction à l'encontre du perturbateur. Plus le désordre causé est grand, plus grande sera la sanction. La maladie dans ce contexte est alors vécue comme un châtiment ${ }^{21}$ en vue de rappeler aux mortels leur condition.

L'autorisation de se rendre dans les carrières, est assujettie à des jours spécifiques marqués du sceau de jours fastes, où l'argile peut être extraite, ou de jours néfastes, où il est interdit de cueillir l'argile ${ }^{22}$. Les jours fastes opi et ogrebô, sont réservés, dans la cosmogonie Gwa, aux activités de subsistance (pêche, agriculture, etc.). Ils ne correspondent pas à des jours fixes mais sont plutôt comme les jours néfastes déterminés selon les astres, par exemple l'apparition de la lune ${ }^{23}$. Les jours néfastes sont quant à eux dits odissa et oguin. Tous ces interdits, est-il besoin de le rappeler, se justifient dans la mesure où, l'argile est considérée comme un génie et elle doit être de ce fait respectée. Les interdits permettent aussi de garantir l'intégrité du génie qu'elle incarne et de protéger la profession des potières et du succès qu'elles espèrent pour les œuvres qu'elles accouchent avec le concours de ces génies ${ }^{24}$.

Aussi une potière ne peut-elle se rendre dans la carrière d'argile, ni s'adonner au façonnage des récipients si elle a entretenu la veille des rapports sexuels. Elles sont obligées de respecter ces restrictions de leur métier, tout comme dans le cas d'une potière qui n'a pas fini d'observer sa période de veuvage, sous peine de malaises mortels ${ }^{25}$. La phase active de production des poteries, à certains moments, est également frappée d'interdits, d'un ensemble de rites, que la potière doit respecter dans le souci d'éviter la rupture entre elle et la nature, qu'elle considère comme un partenaire divin et géniteur ${ }^{26}$.

Dans la panoplie des situations que nous pouvons qualifier de cas de force majeure, on note qu'une fois que l'argile est extraite et emmenée au village, il n'y a plus d'interdits à observer $^{27}$. Cependant lorsqu'il y a un décès dans le village, il ne peut y avoir de façonnage. Il faut que l'enterrement et les funérailles soient achevés pour autoriser la reprise du travail ${ }^{28}$.

21 Le non-respect des interdits entraine pour les potières l'échec, surtout lors de la cuisson, lorsque certaines céramiques se cassent. D'où le respect scrupuleux des rites et interdits au risque de provoquer la colère des ancêtres, dépositaires du savoir et de la technique. Leur intermédiaire avec le commun, ici la potière, peut être traitée par ces forces invisibles qui gouvernent le monde des vivants, comme indigne de poursuivre l'œuvre dont elle constitue pour les générations futures la courroie de transmission.

\section{Conclusion}

Cette enquête sur les rites observés par les potières Gwa d'Oguédoumé, au cours de leur travail, montre que la production céramique n'est pas seulement un métier, mais un moment spirituel où les potières honorent, par chaque pas qu'elles posent, la mémoire de leurs ancêtres. Il ressort ceci dit, que la céramique bien que relevant de l'utilitaire permet de saisir l'univers mental et psychologique des sociétés qui la perpétuent. En s'exprimant par l'art, ces femmes ont su trouver le chemin qui convient le mieux à l'accomplissement de la vie individuelle par un métier qui leur est réservé, et dont elles transmettent les règles et les secrets de génération en génération ${ }^{29}$. 
comme nous pouvons ainsi le voir rend compte des sociétés traditionnelles dans ce qu'elles ont d'essentiel c'est-à-dire la sauvegarde de l'âme de la communauté sans laquelle tout devient superficiel.

\section{BIBLIOGRAPHIE}

HAMPATE BA Amadou, Aspects de la civilisation africaine, Paris, Présence africaine, 1972, http:// www.webpulaaku.net/defte/ahb/aspects/rapports.html (Page consultée le 13/08/2012).

BERTAUX Christian, JESPERS Philippe, « Quelques opérations sacrificielles liées aux géomancies bambara et minyanka du Mali », Systèmes de pensée en Afrique noire, $n^{\circ} 5$, 1981, p.71-98.

BONI Tanela, «Femmes en création : la main, l'esprit, la voix », in Femmes « bâtisseurs » d'Afrique, Québec, Musée de la civilisation, 2000, p.183-191.

COQUERY-VIDROVITCH Catherine, «Le régime foncier rural en Afrique noire », in Le BRIS Émile, LE ROY E., LEIMDORFER F. (dir.), Enjeux fonciers en Afrique noire, Paris, ORSTOM, Karthala, 1983, p.65-83.

COULIBALY Sinali, Le pays sénoufo, Abidjan-Dakar, N.E.A., 1978.

GADOU Dakouri Maurice, « La préservation de la biodiversité : les réponses de la tradition religieuse africaine », The African Anthropologist, vol 8, n², 2001, p.178-199.

GUEYE Mamadou, « Transformations sociales et implications culturelles », Ethiopiques, revue socialiste de culture négro-africaine, vol.1, n³-4 (nouvelle série, $\left.\mathrm{n}^{\circ} 34-35\right)$ ), 1983, http:// ethiopiques.refer.sn/spip.php?article930 (Page consultée le 21/06/2012).

IBO Guéhi Jonas, Perceptions et pratiques environnementales en milieu traditionnel africain : l'exemple des sociétés ivoiriennes anciennes, Orstom, GIDIS-CI, Centre de Petit Bassam, 1994.

KONAN Alice, Place de la médecine traditionnelle dans les soins de sante primaires à Abidjan (Côte d'Ivoire), Thèse pour le diplôme d'Etat de Docteur en Médecine, Spécialité Médecine générale, Université Toulouse III-Paul Sabatier, 2012, 106 p.

KOUASSI Sran Franck, «Lancement d'une période de deuil et de purification nationale, le vendredi 17 mars 2012 : les précisions du porte-parole du président de la Commission Dialogue, vérité et réconciliation » Ivoire-Presse, 2012, http://news.abidjan.net/h/428602.html (Page consultée le 08/06/2012)

LOUCOU Jean-Noël, Histoire de la Côte d'Ivoire. La formation peuples, Abidjan, CEDA, 1984.

MAKARIUS Laura Levi, Le sacré et la violation des interdits, Paris, Payot, Collection Science de l'Homme, 1974.

MALAN Djah François, « Religion traditionnelle et gestion durable des ressources floristiques en Cote d'Ivoire : Le cas des Ehotilé, riverains du Parc National des îles Ehotilé ", in Vertigo - La revue en sciences de l'environnement, vol.9, $\mathrm{n}^{\circ} 2,2009$, p.1-11.

NIANGORAN-BOUAH Georges, « Idéologie de l'or chez les Akan de Côte-d'Ivoire et du Ghana », Journal des africanistes, t.48 fasc.1. L'or dans les sociétés Akan, 1978, p.127-140. 
OGANDAGA Ghislain R., Le culte des ancêtres. Anthropologie du sacré. http://religion.mrugala.net/ Divers/Cosmogonies.pdf (Page consultée le 10/07/2012)

SENE Amsata, Les structures anthropologiques de l'imaginaire en Afrique noire traditionnelle ou vers une archétypologie des concepts de pratiques rituelle et de représentations sociales, Thèse de Doctorat de Sociologie de l'Université Pierre Mendes France, 2004.

TRIMUA Christian, « La place du chef traditionnel dans le contexte de la décentralisation », Actes de l'Atelier de Sokodé sur le statut juridique de la chefferie traditionnelle au TOGO: incertitudes et ambiguités, 02-03 Mars 2007, non publié, 19 p.

\section{NOTES}

1. SENE Amsata, Les structures anthropologiques de l'imaginaire en Afrique noire traditionnelle ou vers une archétypologie des concepts de pratiques rituelle et de représentations sociales, Thèse de Doctorat de Sociologie de l'Université Pierre Mendes France, 2004, p.129.

2. NIANGORAN-BOUAH Georges, «Idéologie de l'or chez les Akan de Côte-d'Ivoire et du Ghana », in Journal des africanistes, tome 48 fascicule 1. L'or dans les sociétés Akan, 1978, p.127.

3. Les Gwa ou Mgbatto sont environnés par les Attié au nord, les Ebrié à l'ouest, les Abouré à l'est et au sud. Certains clans ont des liens de parenté avec les Abidji de Gomon, LOUCOU Jean -Noël, Histoire de la Côte d'Ivoire. La formation peuples, Abidjan, CEDA, 1984, p.143.

4. Entretiens avec ODJE Clément, 58 ans, Secrétaire de la chefferie, Oguédoumé le 07 février 2012.

5. HAMPATE BA Amadou, Aspects de la civilisation africaine, Paris, Présence africaine, 1972, http:// www.webpulaaku.net/defte/ahb/aspects/rapports.html (Page consultée le 13/08/2012).

6. COQUERY-VIDROVITCH Catherine, "Le régime foncier rural en Afrique noire", in Le BRIS Émile, LE ROY E., LEIMDORFER F. (dir.), Enjeux fonciers en Afrique noire, Paris, ORSTOM, Karthala, 1983, p.66-67.

7. NIANGORAN-BOUAH Georges, «Idéologie... », op.cit., 1978, p.127 ; GADOU Dakouri Maurice, « La préservation de la biodiversité : les réponses de la tradition religieuse africaine », in The African Anthropologist, vol 8, $\mathrm{n}^{\circ} 2,2001, \mathrm{p} .181-183$.

8. IBO Guéhi Jonas, Perceptions et pratiques environnementales en milieu traditionnel africain : l'exemple des sociétés ivoiriennes anciennes, Orstom, GIDIS-CI, Centre de Petit Bassam, 1994, p.3.

9. GADOU Dakouri Maurice, « La préservation... », op.cit., 2001, p.183-185.

10. COULIBALY Sinali, Le pays sénoufo, Abidjan-Dakar, N.E.A., 1978, p.118.

11. MALAN Djah François, «Religion traditionnelle et gestion durable des ressources floristiques en Cote d'Ivoire : Le cas des Ehotilé, riverains du Parc National des Îles Ehotilé », Vertigo - La revue en sciences de l'environnement, vol.9, $\mathrm{n}^{\circ} 2,2009, \mathrm{p} .2$.

12. GUÈYE Mamadou, «Transformations sociales et implications culturelles », Ethiopiques, revue socialiste de culture négro-africaine, vol.1, n³-4, 1983 (nouvelle série, n³4-35) http:// ethiopiques.refer.sn/spip.php?article930 (Page consultée le 21/06/2012).

13. TRIMUA C., "La place du chef traditionnel dans le contexte de la décentralisation », Actes de l'Atelier de Sokodé sur le statut juridique de la chefferie traditionnelle au TOGO : incertitudes et ambiguités, 02-03 Mars 2007, non publié, p.6.

14. OGANDAGA Ghislain R., Le culte des ancêtres. Anthropologie du sacré. http:// religion.mrugala.net/Divers/Cosmogonies.pdf (Page consultée le 10/07/2012).

15. BERTAUX Christian, JESPERS Philippe, «Quelques opérations sacrificielles liées aux géomancies bambara et minyanka du Mali », Systèmes de pensée en Afrique noire, n5, 1981, p.71.

16. HAMPATE BA Amadou, Aspects..., op.cit., 1972 ; KOUASSI Sran Franck, 2012, « Lancement d'une période de deuil et de purification nationale, le vendredi 17 mars 2012 : les précisions du porte- 
parole du président de la Commission Dialogue, vérité et réconciliation ", Ivoire-Presse, 8 mars 2012, http://news.abidjan.net/h/428602.html (Page consultée le 08/06/2012).

17. Entretiens avec BOUAME Youa Félicité, 67 ans, Potière, Oguédoumé le 06 février 2012.

18. Entretiens avec KOFFI Atoua, 68 ans, Potière, Oguédoumé le 07 février 2012.

19. MAKARIUS Laura Levi, Le sacré et la violation des interdits, Paris, Payot, 1974, p.18 et p.20-21.

20. Entretiens avec ODJE Clément, 58 ans, Secrétaire de la chefferie, Oguédoumé le 07 février 2012.

21. KONAN Alice, Place de la médecine traditionnelle dans les soins de santé primaires à Abidjan (Côte d'Ivoire), Thèse pour le diplôme d'Etat de Docteur en Médecine Spécialité Médecine générale, Université Toulouse III-Paul Sabatier, 2012, p.13.

22. BONI Tanela, « Femmes en création : la main, l'esprit, la voix », Femmes " bâtisseurs » d'Afrique, Québec, Musée de la civilisation, 2000, p.184-185.

23. Entretiens avec ODJE Clément, 58 ans, Secrétaire de la chefferie, Oguédoumé le 07 février 2012.

24. Ibidem

25. Entretiens avec BOUAME Youa Félicité, 67 ans, Potière, Oguédoumé le 06 février 2012.

26. GADOU Dakouri Maurice, «La préservation... », op.cit., 2001, p.186

27. Entretiens avec KOFFI Atoua, 68 ans, Potière, Oguédoumé le 07 février 2012.

28. Entretiens avec BOUAME Youa Félicité, 67 ans, Potière, Oguédoumé le 06 février 2012.

29. BONI Tanela, « Femmes en création... », op.cit., p.184.

\section{RÉSUMÉS}

Le rite s'appréhende comme un acte à caractère répétitif, des gestes, des paroles ou des attitudes adaptées à une circonstance ou à une finalité désirée. Nous insistons, à travers des enquêtes de terrain, sur les volets transmission et pérennisation des savoir-faire céramique, de la conception à la création, chez les Gwa. Ce peuple forestier de Côte d'Ivoire est l'un des rares où l'on puisse trouver des potières, qui se sont adonnées, dans un passé récent de façon active, à la production céramique. C'est donc le rite en tant qu'idée, conception et courant d'initiation, conduisant à l'action ; pérennisation, par la création matérielle au bout de la chaîne née d'une pratique traditionnelle et technique ; que nous élucidons à travers un exemple ivoirien.

The rite is apprehended as an act of a repetitive characters, gestures, words or attitudes appropriate to a circumstance or a desired purpose. Weinsist, through field surveys, on transmission and sustainability aspects of ceramic know-how, from conception to creation, among Gwa. This forest population of the Ivory Coast is one of the few where we can find potters, who, in recent past, have consecrated their activity to the ceramic production. Rite is thus an idea a conception, leading to an action ; sustainability through material creation at the end of the chain, arisen from a traditional and technical practice, which we clarify through an Ivorian example. 


\section{INDEX}

Mots-clés : histoire des techniques, époque contemporaine, côte d'ivoire, potière, céramique, rites

Index géographique : Côte d'Ivoire

Index chronologique : Époque contemporaine

Thèmes : Horizons internationaux

Keywords : history of technology, contemporary time, Ivory Coast, potter, ceramic, rites.

\section{AUTEUR}

\section{SIMÉON KOUAKOU KOUASSI}

Kouakou Siméon KOUASSI, est Maître de Conférences d'Archéologie à l'Université Félix Houphouët-Boigny de Cocody-Abidjan. Ces travaux portent sur les amas coquilliers, la céramique et les us et coutumes de la zone côtière et forestière de Côte d'Ivoire. Il est le coordonnateur de l'équipe de recherche Céramique, Société et Patrimoine (CSP) de l'Institut des Sciences Anthropologiques de Développement (ISAD) où il est en poste. Il y assure, par ailleurs, la fonction de Chef de l'Unité Pédagogique Archéologie. 